\title{
Usabilidade e acessibilidade: heurísticas de usabilidade em projetos destinados a pessoas com deficiência
}

\author{
Usability and accessibility: usability heuristics in projects for people \\ with disabilities
}

GODOY, Lígia de

Universidade do Estado de Santa Catarina - UDESC I ligiadegodoy@gmail.com

FERREIRA, Marcelo Gitirana Gomes

Universidade do Estado de Santa Catarina - UDESC I marcelo.gitirana@gmail.com

CINELLI, Milton José

Universidade do Estado de Santa Catarina - UDESC I milton.cinelli@udesc.br

\begin{abstract}
Resumo
A pesquisa buscou analisar a utilização de heurísticas de usabilidade em projetos voltados a pessoas com deficiência, investigando a existência de heurísticas específicas com esse objetivo. Através de uma revisão bibliográfica, demonstrou-se a importância da coordenação entre as heurísticas de usabilidade e as diretrizes de acessibilidade, contando com poucos resultados em que isso se concretizou. Estudos futuros poderão aprofundar a pesquisa e, possivelmente, desenvolver heurísticas de usabilidade destinadas a

\section{Abstract}

The aim of this research is to analyze the use of usability heuristics in projects for people with disabilities, investigating the existence of specific heuristics for this purpose. A bibliographic review showed the importance of the coordination between usability heuristics and accessibility guidelines - which few results succeeded to meet. Future studies could help expanding the research and enable the development of usability heuristics for accessible projects.
\end{abstract} projetos acessíveis.

Palavras-chave: Usabilidade. Heurísticas. Diretrizes.

Keywords: Usability. Heuristics. Guidelines. Accessibility. 


\section{INTRODUC̣ÃO}

A usabilidade, ainda que não se limite a esta área, está originalmente ligada à Interação Humano-Computador (IHC), podendo ser entendida como o grau de facilidade com que uma interface responde à interação com o usuário. A interface é considerada em um sentido amplo, podendo fazer parte de uma máquina, um website, um dispositivo eletrônico, entre outros. Como facilitadoras do projeto de interfaces que contemplem uma boa usabilidade, foram criadas as heurísticas, que são diretrizes que podem servir de base tanto ao desenvolvimento, quanto à avaliação de usabilidade de interfaces. Além da usabilidade, outro requisito ao qual os projetistas devem atentar é a acessibilidade. Em relação às interfaces web percebe-se uma atenção crescente à acessibilidade, contando inclusive com diretrizes, internacionais e nacionais, para que esteja garantida. A acessibilidade busca prover o acesso às informações a todas as pessoas, independentemente de suas deficiências, não devendo, porém, ser confundida com a usabilidade. Ainda que uma interface seja acessível a pessoas com deficiência, ela pode apresentar problemas de usabilidade, comprometendo o desempenho da tarefa ou a satisfação do usuário. Da mesma forma, projetar uma interface para a usabilidade não garante que essa será acessível a pessoas com deficiência. Partindo desse contexto, o presente artigo analisa heurísticas de usabilidade utilizadas no desenvolvimento e na avaliação de projetos acessíveis a pessoas com deficiência. Busca-se, dessa forma, investigar de que maneira é conduzida a integração entre essas duas premissas, a acessibilidade e a usabilidade, as diretrizes e heurísticas utilizadas em cada caso, e a existência de heurísticas específicas voltadas à usabilidade de projetos acessíveis.

\section{USABILIDADE E HEURÍSTICAS}

A usabilidade é definida pela ISO 9241-11 (ABNT, 2002) como sendo a medida pela qual um produto pode ser utilizado por usuários específicos para alcançar objetivos específicos com eficácia, eficiência e satisfação em um contexto específico de uso. Ou, de acordo com a definição de Rubin e Chisnell (2008), a usabilidade é tida como ausência de frustração, sendo atingida quando o usuário pode fazer o que quer, da maneira que espera ser capaz de fazer, sem obstáculos, hesitações ou dúvidas. Os autores apontam algumas premissas para a usabilidade, a saber: utilidade, eficiência, eficácia, capacidade de aprendizagem (learnability), satisfação e acessibilidade. A utilidade compreende o grau em que um produto permite ao usuário atingir os seus objetivos. A eficiência indica a rapidez com que os objetivos do usuário podem ser alcançados de forma precisa e completa, e geralmente é uma medida de tempo. Já a eficácia significa a extensão na qual o produto se comporta da forma como o usuário espera e a facilidade de uso, sendo normalmente mensurada 
quantitativamente, através de taxas de erro. A capacidade de aprendizagem parte da eficácia e indica a habilidade do usuário de operar o sistema com um determinado nível de competência, após um determinado tempo de treinamento, ou tempo algum. A satisfação refere-se às percepções, sentimentos e opiniões do usuário sobre o produto e a acessibilidade busca promover o acesso aos produtos necessários para cumprir um determinado objetivo, e será melhor discutida no capítulo seguinte. Ao comparar ambas as definições, aquela que consta na ISO 9241-11 (ABNT, 2002) e a dos autores Rubin e Chisnell (2008), percebe-se, conforme apresentado na Figura 1, que as definições são similares, porém Rubin e Chisnell (2008) abarcam mais premissas do que a ISO 9241-11 (ABNT, 2002), oferecendo uma definição mais abrangente. Apesar de Rubin e Chisnell (2008) não mencionarem o contexto de uso nessa definição principal, como acontece na ISO 9241-11 (2002), os autores o consideram como um dos três componentes principais em qualquer situação de desempenho humano, junto com o usuário e a atividade.

Figura 1 - Comparação entre as definições de usabilidade.

\section{Definição de Usabilidade ISO 9241-11 (1998):}

\section{Definição de Usabilidade Rubin e Chisnell (2008):}

\section{Medida pela qual um produto pode ser utilizado por usuários específicos para alcançar objetivos específicos com eficácia, eficiência e satisfação em um contexto específico de uso.}

\begin{tabular}{|l|l|}
\hline Premissas: & Premissas: \\
\hline - Eficácia & - Eficácia \\
\hline - Eficiência & - Eficiência \\
\hline - Satisfação & - Satisfação \\
\hline & - Utilidade \\
\hline & - Capacidade de aprendizagem \\
\hline
\end{tabular}

Fonte: Os autores.
Atingida quando o usuário pode fazer o que quer, da maneira que espera ser capaz de fazer, sem obstáculos, hesitações ou dúvidas.
Premissas:

Eficácia

- Satisfação

- Utilidade

Capacidade de aprendizagem

essibilidade

Cybis, Betiol e Faust (2015) afirmam que, apesar de a construção de um sistema com usabilidade demandar uma análise cuidadosa de uma série de componentes, existe o que os autores chamam de "configuração de base", que pode ser seguida para favorecer o estabelecimento da usabilidade no sistema. Essa configuração é facilitada por critérios, diretrizes ou heurísticas de usabilidade, propostos por diversos autores. Entre as heurísticas de usabilidade mais difundidas estão as dez heurísticas propostas por Jakob Nielsen (1994) e as chamadas "regras de ouro", de Shneiderman e Plaisant (2005), ambas listadas no Quadro 1, de forma comparativa, ou seja, apontando as equivalências existentes entre as heurísticas dos dois autores. 
Quadro 1 - Comparação entre as Heurísticas de Nielsen e Regras de Ouro de Shneiderman e Plaisant (2005). O símbolo - indica a inexistência da heurística correspondente do autor.

\begin{tabular}{l|l}
\hline Heurísticas de Nielsen (1995) & $\begin{array}{l}\text { Regras de Ouro } \\
\text { (SHNEIDERMAN; PLAISANT, 2005) }\end{array}$ \\
\hline $\begin{array}{l}\text { Visibilidade do estado do sistema (feedback) } \\
\text { Liberdade e controle ao usuário }\end{array}$ & $\begin{array}{l}\text { Oferecer feedback informativo } \\
\text { Suportar o usuário como controlador } \\
\text { Consistência e padrões }\end{array}$ \\
$\begin{array}{l}\text { Perseguir a consistência } \\
\text { Prevenção de erros }\end{array}$ & $\begin{array}{l}\text { Reduzir a carga de memória de curto prazo } \\
\text { Reconhecer em vez de relembrar }\end{array}$ \\
$\begin{array}{l}\text { Flexibilidade e eficiência de uso } \\
\text { as necessidades de usuários diversos) }\end{array}$ \\
$\begin{array}{l}\text { Correspondência entre o sistema e o mundo } \\
\text { real }\end{array}$ & - \\
Design estético e minimalista & - \\
$\begin{array}{l}\text { Suporte para o usuário reconhecer, } \\
\text { diagnosticar e recuperar erros }\end{array}$ & - \\
Ajuda e documentação & Permitir fácil reversão de ações \\
\hline
\end{tabular}

Fonte: Os autores.

\section{ACESSIBILIDADE, DIRETRIZES E SUA RELAC̣ÃO COM A USABILIDADE}

A acessibilidade busca assegurar o acesso de pessoas com deficiência, em igualdade de oportunidades, a espaços físicos, produtos e informações, inclusive no meio digital. A acessibilidade no meio eletrônico é regida por algumas diretrizes, em especial as Diretrizes de Acessibilidade para o Conteúdo da Web - WCAG (Web Content Accessibility Guidelines). As WCAG, apresentadas no Quadro 2, foram desenvolvidas com o objetivo de fornecer um padrão comum para acessibilidade de conteúdo na web, que atenda às necessidades dos indivíduos, organizações e governos ao redor do mundo (W3, 2017). As WCAG foram atualizadas em 2008, e as WCAG 2.0, versão mais atual, foram aprovadas como uma norma ISO e são divididas em quatro princípios: perceptível, operacional, compreensível e robusto. Outro conjunto de diretrizes para acessibilidade está compreendido na Seção 508 da Lei de Reabilitação americana (Rehabilitation Act) de 1973, voltada à acessibilidade de tecnologias de informação em meio eletrônico desenvolvidas, adquiridas, mantidas ou utilizadas por agências do governo federal americano (GSA, 2017). Similar à Seção 508, o Brasil conta com o e-MAG (Modelo de Acessibilidade em Governo Eletrônico), que está alinhado às recomendações internacionais 
e busca facilitar o acesso de todas as pessoas às informações e serviços disponibilizados nos websites e portais do governo. O e-MAG traz algumas recomendações para desenvolvedores, baseadas também nas WCAG 2.0.

Quadro 2 - Diretrizes de Acessibilidade para o Conteúdo da Web - WCAG.

\begin{abstract}
Princípio 1: Perceptível - Os componentes da informação e da interface devem ser apresentados aos usuários de forma que eles possam percebê-los.
\end{abstract}

Diretriz 1.1: Alternativas de texto - Forneça alternativas de texto para qualquer conteúdo que não seja de texto, de modo que ele possa ser alterado para outros formatos, como impressão em tamanhos grandes, braile, fala, símbolos ou linguagem mais simples.

Diretriz 1.2: Mídia dinâmica ou contínua - Forneça alternativas para conteúdo em mídia dinâmica ou temporal.

Diretriz 1.3: Adaptável - Crie conteúdo que possa ser apresentado de maneiras diferentes (por exemplo, layout mais simples) sem perder informações ou estrutura.

Diretriz 1.4: Distinguível - Facilite aos usuários ver e ouvir conteúdo, através da separação do primeiro plano do fundo.

Princípio 2: Operacional - Os componentes de interface de usuário e navegação devem ser operacionais

Diretriz 2.1: Teclado acessível - Torne todas as funcionalidades disponíveis a partir de um teclado.

Diretriz 2.2: Tempo suficiente - Forneça aos usuários tempo suficiente para ler e usar o conteúdo.
Diretriz 2.3: Convulsões - Não conceba conteúdo de forma que seja conhecida por causar convulsões.

Diretriz 2.4: Navegável - Forneça maneiras de ajudar os usuários a navegar, encontrar conteúdo e determinar onde eles estão.

Princípio 3: Compreensível - A informação e a operação da interface do usuário devem ser compreensíveis.

Diretriz 3.1: Legível - Torne o conteúdo do texto legível e compreensível.

Diretriz 3.2: Previsível - Faça com que as páginas web apareçam e funcionem de forma previsível.

Diretriz 3.3: Assistência na inserção dos dados - Ajude os usuários a evitar e corrigir erros.

\section{Princípio 4: Robusto}

- O conteúdo deve ser suficientemente robusto para que possa ser interpretado de forma confiável por uma grande variedade de agentes de usuários, incluindo tecnologias de apoio.

Fonte: Adaptado de W3 (2017).
Diretriz 4.1: Compatível - Maximize a compatibilidade com agentes atuais e futuros do usuário, incluindo tecnologias assistivas.

A acessibilidade é citada por Rubin e Chisnell (2008) como um requisito de usabilidade de um produto, junto à utilidade, eficiência, eficácia, satisfação e capacidade de aprendizagem. Os autores tratam a acessibilidade também como uma forma de usabilidade voltada a pessoas com deficiência, ou em contextos especiais. Reforçam que, ao se desenvolver um produto com usabilidade voltado esse público, quase sempre são também beneficiadas pessoas que não fazem parte dele. Percebe-se, portanto, uma preocupação dos autores supracitados em atrelar a acessibilidade à usabilidade, de maneira que uma não possa existir sem a outra. Os autores afirmam que a usabilidade e a acessibilidade estão 
compreendidas em uma disciplina mais abrangente, o "design centrado no usuário", chamado por alguns autores de "design centrado no humano". Segundo Norman (2013), o design centrado no humano é o processo que visa garantir que as necessidades das pessoas sejam satisfeitas, resultando em um produto que ofereça usabilidade e inteligibilidade, e que cumpra as tarefas desejadas, trazendo uma experiência de uso positiva e agradável. O design centrado no humano seria, de acordo com o autor, uma forma de abranger uma série de requisitos de projeto, com foco em duas coisas: resolver o problema certo, e fazê-lo de forma a atender às necessidades e capacidades humanas. Rubin e Chisnell (2008) posicionam o design centrado no usuário, ou no humano, como parte de um conceito ainda mais amplo, chamado "design de experiência". O "design de experiência" engloba, como o próprio nome sugere, a experiência do usuário durante toda a sua interação com o produto ou serviço, não apenas no momento da compra ou de utilização.

\section{MÉTODO}

A pesquisa foi realizada por meio de um levantamento bibliográfico feito nas bases de dados Scopus e Scielo, consideradas duas fontes relevantes e que englobam outras bases de dados. As pesquisas foram limitadas a artigos de periódicos, não sendo limitadas em relação à data da publicação ou à área de estudo. Foram utilizados como critérios de inclusão a disponibilidade dos artigos por meio de acesso na universidade, de forma gratuita, e o fato de serem artigos primários, excluindo-se artigos de revisão bibliográfica, que, caso trouxessem contribuições à pesquisa, foram incluídos no referencial teórico. A busca limitou-se também a artigos nos idiomas inglês, português e espanhol, idiomas de domínio dos autores. A construção dos argumentos de busca procurou abranger as publicações que tratassem de heurísticas de usabilidade em projetos voltados a pessoas com deficiência. Dessa forma, incluíramse, simultaneamente, os termos "heurísticas", "deficiência" e sinônimos, e "usabilidade", na língua inglesa, o que permite encontrar publicações em diversas línguas, por ser comum que essas contenham o resumo também em língua inglesa.

Após a busca nas bases de dados, prosseguiu-se para os filtros. $O$ primeiro filtro consistiu na leitura do título, resumo e palavras-chave, e no segundo filtro procedeu-se à leitura das publicações completas, o que resultou na lista final de artigos. A lista final conta com 11 artigos, 10 deles provenientes da base Scopus e um da base Scielo. Um dos artigos da lista final, de Leporini e Paternò (2003) é uma publicação proveniente de evento, e não de periódico, e foi incluído manualmente por ser considerado relevante, já que está relacionado a um artigo dos mesmos autores que constava na pesquisa, porém ao qual não se teve acesso. Buscou-se analisar as publicações quanto à existência ou à utilização de heurísticas de usabilidade específicas para pessoas com 
deficiência, bem como a utilização de diretrizes de usabilidade e acessibilidade, e a forma como ambas, acessibilidade e usabilidade, foram tratadas. As questões de pesquisa formuladas para essa análise foram as seguintes:

- O artigo utiliza heurísticas/diretrizes de usabilidade voltadas a pessoas com deficiência? Quais?

- O artigo utiliza heurísticas/diretrizes específicas de usabilidade? Quais? - O artigo utiliza heurísticas/diretrizes específicas de acessibilidade? Quais? As publicações também foram analisadas quanto ao objetivo, para melhor compreensão da aplicação das diretrizes de acessibilidade e heurísticas de usabilidade.

\section{RESULTADOS E DISCUSSÃO}

Os resultados da pesquisa foram apresentados em dois quadros. Ainda que a pesquisa não tenha sido limitada quanto à data de publicação dos artigos, os resultados retornaram publicações recentes, com o maior número de publicações em 2017, como se pode perceber na Figura 2. O Quadro 3 elenca as publicações levantadas, bem como os autores e objetivo de cada uma delas. Ainda que a pesquisa não tenha sido limitada em relação ao meio, físico ou digital, percebe-se uma predominância de publicações voltadas a interfaces digitais, especialmente ao desenvolvimento de websites e softwares, tendo apenas uma das publicações, de Mi et al. (2014), levado em consideração a interface física do produto estudado.

Figura 2 - Número de publicações ao longo do tempo.

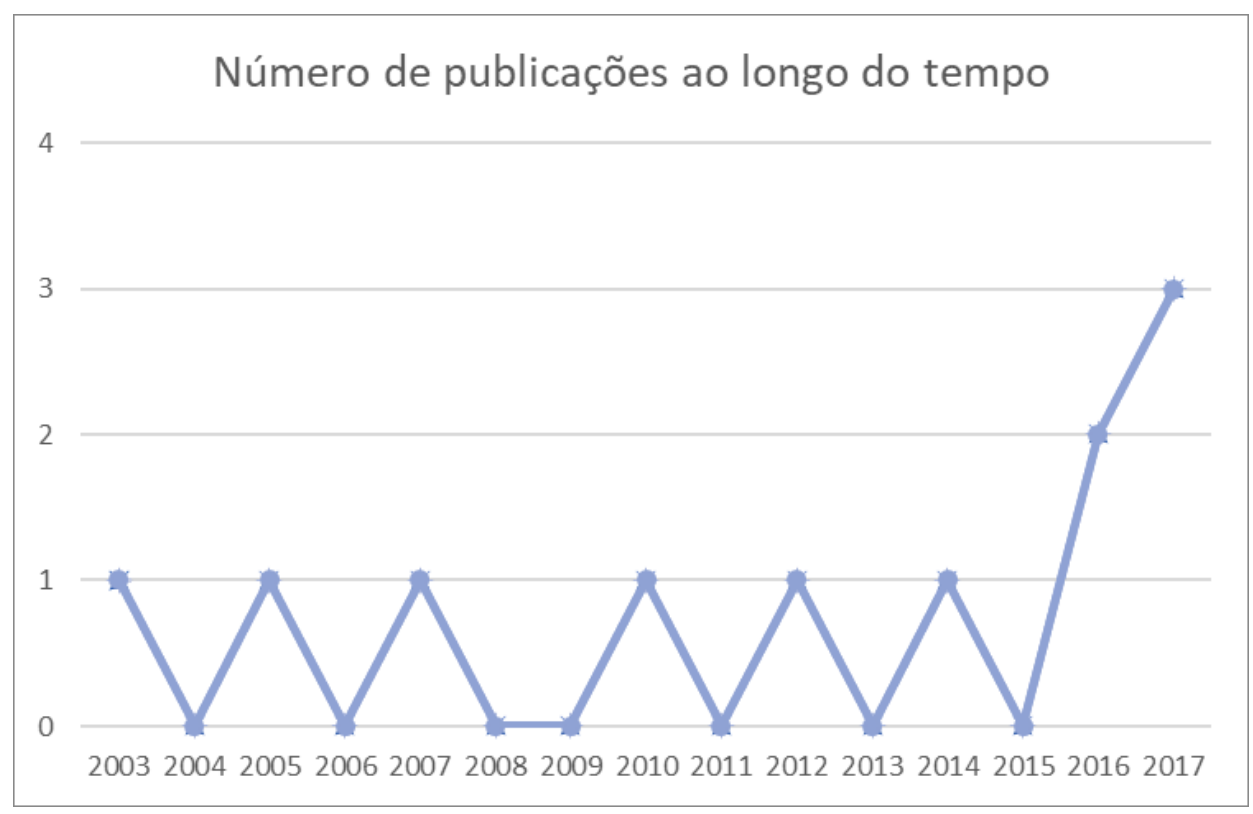

Fonte: Os autores. 
Quadro 3 - Publicações analisadas e objetivos.

\begin{tabular}{|c|c|c|}
\hline Referência & Título & Objetivo \\
\hline (Ml et al., 2014) & $\begin{array}{l}\text { A Heuristic Checklist } \\
\text { for an Accessible } \\
\text { Smartphone Interface } \\
\text { Design }\end{array}$ & $\begin{array}{l}\text { Propor e empregar um método para analisar } \\
\text { as diretrizes de projeto existentes, validando- } \\
\text { as com a entrada do usuário e desenvolvendo } \\
\text { uma lista de verificação heurística para uso por } \\
\text { desenvolvedores de dispositivos móveis. }\end{array}$ \\
\hline $\begin{array}{l}\text { (STEWART; } \\
\text { NARENDRA, } \\
\text { 2005) }\end{array}$ & $\begin{array}{l}\text { Accessibility and } \\
\text { Usability of Online } \\
\text { Library Databases }\end{array}$ & $\begin{array}{l}\text { Investigar a acessibilidade e a usabilidade das } \\
\text { bibliotecas acadêmicas ou bases de dados } \\
\text { online para pessoas que utilizam softwares } \\
\text { adaptativos para interagir com computadores. }\end{array}$ \\
\hline $\begin{array}{l}\text { (HARNISS et al., } \\
\text { 2007) }\end{array}$ & $\begin{array}{l}\text { Considerations for } \\
\text { Developing Interfaces } \\
\text { for Collecting } \\
\text { Patient-Reported } \\
\text { Outcomes That } \\
\text { Allow the Inclusion } \\
\text { of Individuals With } \\
\text { Disabilities }\end{array}$ & $\begin{array}{l}\text { Descrever um sistema para coleta de } \\
\text { resultados relatados por pacientes (PROMIS), } \\
\text { mencionando seus testes de usabilidade e } \\
\text { acessibilidade, com usuários sem nenhuma } \\
\text { deficiência e também com pessoas com } \\
\text { deficiência. }\end{array}$ \\
\hline $\begin{array}{l}\text { (YADRICH et al., } \\
\text { 2012) }\end{array}$ & $\begin{array}{l}\text { Creating Patient and } \\
\text { Family Education } \\
\text { Web Sites: Assuring } \\
\text { Accessibility and } \\
\text { Usability Standards }\end{array}$ & $\begin{array}{l}\text { Relatar os métodos e processos utilizados } \\
\text { para garantir que a usabilidade e a } \\
\text { acessibilidade tenham sido alcançadas durante } \\
\text { o desenvolvimento de um website para } \\
\text { cuidadores e membros da família de pacientes } \\
\text { com doenças crônicas. }\end{array}$ \\
\hline $\begin{array}{l}\text { (LEPORINI; } \\
\text { PATERNÒ, 2003) }\end{array}$ & $\begin{array}{l}\text { Criteria for Usability } \\
\text { of Accessible Web } \\
\text { Sites }\end{array}$ & $\begin{array}{l}\text { Identificar as relações entre os dois conceitos, } \\
\text { usabilidade e acessibilidade, e propor um } \\
\text { conjunto de critérios de usabilidade para } \\
\text { websites acessíveis, com o objetivo de melhorar } \\
\text { a navegabilidade para usuários com deficiência. }\end{array}$ \\
\hline $\begin{array}{l}\text { (KING; } \\
\text { YOUNGBLOOD, } \\
\text { 2016) }\end{array}$ & $\begin{array}{l}\text { E-Government } \\
\text { in Alabama: } \\
\text { An Analysis of } \\
\text { County Voting and } \\
\text { Election Website } \\
\text { Content, Usability, } \\
\text { Accessibility, and } \\
\text { Mobile Readiness }\end{array}$ & $\begin{array}{l}\text { Analisar a qualidade de informação, } \\
\text { acessibilidade, usabilidade e adequação para } \\
\text { dispositivos móveis de websites de eleição no } \\
\text { estado do Alabama/EUA. }\end{array}$ \\
\hline $\begin{array}{l}\text { (ALI; ALBALUSHI; } \\
\text { ALBADI, 2017) }\end{array}$ & $\begin{array}{l}\text { Guidelines and } \\
\text { Deployment of } \\
\text { Accessibility-Aware } \\
\text { Framework Approach }\end{array}$ & $\begin{array}{l}\text { Facilitar o pleno acesso à web por pessoas com } \\
\text { deficiência, através de diretrizes que fornecem } \\
\text { a base para a construção de uma abordagem } \\
\text { estrutural consciente da acessibilidade. }\end{array}$ \\
\hline $\begin{array}{l}\text { (ANSARI et al., } \\
\text { 2016) }\end{array}$ & $\begin{array}{l}\text { Heuristic, } \\
\text { Accessibility and } \\
\text { Usability Evaluations } \\
\text { of Pakistan's } \\
\text { E-Government } \\
\text { Websites }\end{array}$ & $\begin{array}{l}\text { Avaliar as heurísticas, a acessibilidade e } \\
\text { usabilidade enfrentadas por cidadãos em } \\
\text { websites do governo. }\end{array}$ \\
\hline $\begin{array}{l}\text { (SCHMUTZ; } \\
\text { SONDEREGGER; } \\
\text { SAUER, 2017) }\end{array}$ & $\begin{array}{l}\text { Implementing } \\
\text { Recommendations } \\
\text { From Web } \\
\text { Accessibility } \\
\text { Guidelines: A } \\
\text { Comparative Study } \\
\text { of Nondisabled Users } \\
\text { and Users With } \\
\text { Visual Impairments }\end{array}$ & $\begin{array}{l}\text { Examinar se a implementação das } \\
\text { recomendações das diretrizes de acessibilidade } \\
\text { na web teria diferentes efeitos entre pessoas } \\
\text { sem nenhuma deficiência e pessoas com } \\
\text { deficiência visual. }\end{array}$ \\
\hline
\end{tabular}




\begin{tabular}{|c|c|c|}
\hline $\begin{array}{l}\text { (PICKING et al., } \\
2010)\end{array}$ & $\begin{array}{l}\text { Simplicity, } \\
\text { Consistency, } \\
\text { Universality, } \\
\text { Flexibility and } \\
\text { Familiarity }\end{array}$ & $\begin{array}{l}\text { Descrever o projeto da interface, e a } \\
\text { subsequente avaliação de usabilidade } \\
\text { do projeto Easyline+, que envolveu o } \\
\text { desenvolvimento de tecnologia assistiva } \\
\text { ambiente para auxiliar idosos e pessoas com } \\
\text { deficiência nas suas interações com utensílios } \\
\text { de cozinha. }\end{array}$ \\
\hline $\begin{array}{l}\text { (ALVAREZ; } \\
\text { JARAMILLO; } \\
\text { BUILES, 2017) }\end{array}$ & $\begin{array}{l}\text { Evaluación Heurística } \\
\text { de la Usabilidad } \\
\text { de Software para } \\
\text { Facilitar el Uso } \\
\text { del Computador } \\
\text { a Personas en } \\
\text { Situación de } \\
\text { Discapacidad Motriz }\end{array}$ & $\begin{array}{l}\text { Propor um método com enfoque heurístico } \\
\text { para avaliar a usabilidade de um software } \\
\text { desenvolvido para facilitar o acesso à } \\
\text { computação a pessoas com deficiência motriz. }\end{array}$ \\
\hline
\end{tabular}

Fonte: Os autores.

O Quadro 4 sistematiza a análise das publicações, realizada a partir das perguntas de pesquisa previamente definidas, de modo a facilitar a discussão posterior. Pode-se, dessa forma, visualizar quais publicações trouxeram heurísticas de usabilidade destinadas a projetos acessíveis e quais utilizaram heurísticas ou diretrizes de acessibilidade e/ou usabilidade de forma separada.

Quadro 4 - Análise das publicações.

\begin{tabular}{|c|c|c|c|}
\hline Publicação & $\begin{array}{l}\text { Utiliza heurísticas/ } \\
\text { diretrizes de } \\
\text { usabilidade voltadas } \\
\text { a pessoas com } \\
\text { deficiência? Quais? }\end{array}$ & $\begin{array}{l}\text { Utiliza heurísticas/ } \\
\text { diretrizes específicas de } \\
\text { usabilidade? } \\
\text { Quais? }\end{array}$ & $\begin{array}{l}\text { Utiliza heurísticas/ } \\
\text { diretrizes específicas } \\
\text { de acessibilidade? } \\
\text { Quais? }\end{array}$ \\
\hline (Ml et al., 2014) & $\begin{array}{l}\text { Sim. Propostas pelos } \\
\text { autores. }\end{array}$ & Não & Não \\
\hline $\begin{array}{l}\text { (STEWART; } \\
\text { NARENDRA, } \\
\text { 2005) }\end{array}$ & Não & $\begin{array}{l}\text { Não. Consideram a } \\
\text { completude das tarefas } \\
\text { como requisito de } \\
\text { usabilidade. }\end{array}$ & $\begin{array}{l}\text { Sim. Compostas pela } \\
\text { maioria das diretrizes } \\
\text { do Section } 508 \text { e Web } \\
\text { Content Accessibility } \\
\text { Guidelines. }\end{array}$ \\
\hline $\begin{array}{l}\text { (HARNISS et al., } \\
\text { 2007) }\end{array}$ & Não & $\begin{array}{l}\text { Não mencionam. } \\
\text { Avaliam a usabilidade } \\
\text { através de auto relatos, } \\
\text { observações, gravações e } \\
\text { entrevistas cognitivas. }\end{array}$ & Sim. Section 508. \\
\hline $\begin{array}{l}\text { (YADRICH et al., } \\
\text { 2012) }\end{array}$ & Não & $\begin{array}{l}\text { Sim. Usability Guidelines } \\
\text { for User-Centered } \\
\text { Website Design, } \\
\text { propostas pelo US } \\
\text { Department of Health } \\
\text { and Human Services } \\
\text { (USDHHS): } 18 \text { diretrizes } \\
\text { mais duas criadas pelos } \\
\text { autores. }\end{array}$ & Sim. Section 508. \\
\hline $\begin{array}{l}\text { (LEPORINI; } \\
\text { PATERNÒ, } \\
\text { 2003) }\end{array}$ & $\begin{array}{l}\text { Sim. Propostas pelos } \\
\text { autores. }\end{array}$ & Não & Não \\
\hline $\begin{array}{l}\text { (KING; } \\
\text { YOUNGBLOOD, } \\
\text { 2016) }\end{array}$ & Não & $\begin{array}{l}\text { Heurísticas construídas } \\
\text { pelos autores com base } \\
\text { em trabalhos anteriores. }\end{array}$ & $\begin{array}{l}\text { Sim. Section } 508 \\
\text { e Web Content } \\
\text { Accessibility } \\
\text { Guidelines 2.0. }\end{array}$ \\
\hline
\end{tabular}


GODOY, Lígia de; FERREIRA, Marcelo Giritana Gomes; CINELLI, Milton José

\begin{tabular}{|c|c|c|c|}
\hline $\begin{array}{l}\text { (ALI; } \\
\text { ALBALUSHI; } \\
\text { ALBADI, 2017) }\end{array}$ & Não & Não & $\begin{array}{l}\text { Sim. Propostas pelos } \\
\text { autores }\end{array}$ \\
\hline $\begin{array}{l}\text { (ANSARI et al., } \\
\text { 2016) }\end{array}$ & Não & $\begin{array}{l}\text { Sim. Heurísticas de Jakob } \\
\text { Nielsen. }\end{array}$ & $\begin{array}{l}\text { Sim. Web Content } \\
\text { Accessibility } \\
\text { Guidelines 2.0. }\end{array}$ \\
\hline $\begin{array}{l}\text { (SCHMUTZ; } \\
\text { SONDEREGGER; } \\
\text { SAUER, 2017) }\end{array}$ & Não & Não mencionam. & $\begin{array}{l}\text { Sim. Web Content } \\
\text { Accessibility } \\
\text { Guidelines 2.0. }\end{array}$ \\
\hline $\begin{array}{l}\text { (PICKING et al., } \\
\text { 2010) }\end{array}$ & Não & $\begin{array}{l}\text { Sim. Heurísticas } \\
\text { propostas pelos autores } \\
\text { (SCUFF). }\end{array}$ & Não \\
\hline $\begin{array}{l}\text { (ALVAREZ; } \\
\text { JARAMILLO; } \\
\text { BUILES, 2017) }\end{array}$ & $\begin{array}{l}\text { Sim. Propostas pelos } \\
\text { autores. }\end{array}$ & Não & Não \\
\hline
\end{tabular}

Fonte: Os autores.

Mi et al. (2014) propõem um conjunto de heurísticas especificamente destinadas ao projeto de smartphones acessíveis, compreendendo no estudo tanto as interfaces virtuais como as interfaces físicas destes. Os autores, partindo da literatura, compuseram um conjunto de diretrizes, que foi testado a partir de uma avaliação heurística de protótipos construídos para este fim. Mi et al. (2014) demonstram preocupação com a usabilidade, sendo realizada uma avaliação de usabilidade dos protótipos com usuários. A avaliação de usabilidade foi chamada pelos autores de usable accessibility testing, o que pode ser compreendido como um teste que avalia as duas premissas, usabilidade e acessibilidade, de forma articulada. Mi et al. (2004) apontam a carência, na literatura sobre Interação Humano-Computador (IHC), de parâmetros e definições padronizados relacionados à acessibilidade de dispositivos móveis (mobile) e à relação entre usabilidade e acessibilidade. Seguindo o mesmo caminho, Stewart e Narendra (2005), assim como outros autores, atentam para o fato de que o atendimento a padrões e diretrizes de acessibilidade não necessariamente conduz à usabilidade de um projeto. Os autores realizaram uma primeira avaliação de adequação de bases de dados virtuais às diretrizes de acessibilidade, para então avaliar o que chamam de usabilidade funcional, ou funcionalidade, através de testes com usuários que utilizam tecnologias assistivas. Leporini e Paternò (2003) buscam, assim como os autores anteriores, compreender as relações entre a acessibilidade e a usabilidade, conceitos que usualmente são tratados separadamente, segundo os autores. Os autores falam sobre a tendência de se considerar apenas questões de acessibilidade, ignorando aquelas relacionadas à usabilidade, quando se trata de websites acessíveis a pessoas com deficiência. Leporini e Paternò (2003) tratam a acessibilidade técnica como uma pré-condição para a usabilidade, reforçando que, mesmo que um website seja teoricamente acessível, por estar em conformidade com os padrões de acessibilidade técnica, ainda pode ser difícil de utilizar por pessoas com deficiência. Atentam para a necessidade de se considerar o contexto no qual os usuários trabalham, como as tecnologias assistivas que suportam suas atividades. No caso da pesquisa, foram considerados usuários que acessam 
websites através de leitores de tela. Leporini e Paternò (2003) demonstram, inclusive, alguns problemas de usabilidade em websites acessíveis, detectados quando os usuários interagem através de um leitor de tela. Na opinião dos autores, as Web Content Accessibility Guidelines - WCAG, conjunto amplamente utilizado por desenvolvedores que buscam a acessibilidade nos projetos, não consideram, de forma satisfatória, a usabilidade nas suas diretrizes. Os autores buscaram, portanto, estender as diretrizes de acessibilidade, visando também melhorar e facilitar o desempenho da tarefa. Partindo dessa premissa, Leporini e Paternò (2003) determinaram 16 diretrizes para melhorar a usabilidade de websites acessíveis para usuários que utilizam leitores de tela.

Harniss et al. (2007) mencionam diretrizes para acessibilidade, não relatando preocupação com as heurísticas ou diretrizes de usabilidade, ainda que mencionem testes de usabilidade. Os autores indicam um protocolo para a realização de testes de usabilidade e acessibilidade que deverão ser aplicados ao desenvolvimento de um sistema que coleta informações relatadas por pacientes. O sistema de informação é destinado a usuários com e sem deficiência, que possam estar utilizando tecnologias assistivas. Os testes seriam realizados através de auto relatos com perguntas abertas, observação e gravação de vídeo por especialistas em usabilidade e acessibilidade e entrevistas cognitivas. A publicação de Yadrich et al. (2012), embora não voltada a pessoas com deficiência, considerou a acessibilidade como requisito no desenvolvimento de um website voltado a cuidadores de pacientes com doenças crônicas. O mesmo ocorreu com King e Youngblood (2016), que consideram a acessibilidade um requisito importante, já que seu estudo é voltado a websites de eleições, devendo ser acessíveis a toda a população em questão. King e Youngblood (2016), assim como Ansari et al. (2016) e Yadrich et al. (2012), utilizam tanto heurísticas de usabilidade quanto as de acessibilidade, porém de forma descoordenada.

Schmutz, Sonderegger e Sauer (2017) realizam uma comparação entre os efeitos da implementação de diretrizes de acessibilidade em usuários que não apresentam nenhuma deficiência e usuários com deficiência visual, reforçando a preocupação com a separação entre acessibilidade e usabilidade e suas consequências. Os resultados dos testes realizados pelos autores indicaram que todos os usuários, independentemente de apresentar alguma deficiência, se beneficiaram de níveis altos de acessibilidade em websites. Em suma, os resultados sugerem que o atendimento aos níveis mais altos de acessibilidade, considerando-se as WCAG 2.0, traria bons efeitos no que concerne à usabilidade. Os autores demonstram a importância desses resultados, principalmente no sentido de mudar a percepção das diretrizes de uma abordagem de "acessibilidade para usuários com deficiência" para uma abordagem de "design inclusivo".

Picking et al. (2010) propõem seu próprio conjunto de heurísticas de usabilidade, a partir de Constantine e Lockwood (1999), Nielsen (1994) e Shneiderman (1999), chamado de SCUFF, acrônimo em inglês para: 
Simplicidade, Consistência, Universalidade, Flexibilidade e Familiaridade. Os autores preocupam-se com a adequação das diretrizes a aplicativos interativos ambientais (ou sistemas ambientais). Mencionam que o sistema pode ser acessível a pessoas com deficiência visual por conta de a interface ser auditiva, não demonstrando, porém, preocupação com diretrizes de usabilidade voltadas especificamente às pessoas com deficiência. Ali, Albalushi e Albadi (2017) atentam para diretrizes de acessibilidade, propondo uma abordagem estrutural que visa guiar os desenvolvedores no projeto de websites voltados a pessoas com deficiência, fornecendo uma fonte única de informação sobre diretrizes e padrões que esses devem seguir. Embora mencionem avaliações de usabilidade, os autores não demonstram preocupação com a utilização de heurísticas para tal, dando maior ênfase às diretrizes de acessibilidade.

Alvarez, Jaramillo e Builes (2017) apontam a possibilidade de se melhorar a acessibilidade de produtos físicos, como teclados e mouses, através de softwares, apresentada como uma alternativa ao enfoque tradicional de criação de produtos para tornar os softwares acessíveis a pessoas com deficiência. Os autores afirmam que a melhor solução para o desenvolvimento de softwares para esse fim é buscar a adaptação das interfaces a necessidades específicas de cada usuário, porém, devido à grande variedade de deficiências e características decorrentes, isso não se dá de forma prática e nem escalável. Alvarez, Jaramillo e Builes (2017) defendem a utilização de uma avaliação heurística, em detrimento de métricas de rendimento e estudos de usabilidade quantitativos, para pessoas com incapacidade motriz. No método proposto, os próprios usuários dos testes de usabilidade classificam as heurísticas, indicando quais são mais importantes, sendo este o grande diferencial, de acordo com os autores.

De modo geral, percebe-se, entre os artigos estudados, uma tendência de se tratar a acessibilidade e a usabilidade de forma independente. Ainda que os autores demonstrem preocupação com ambos os requisitos, parece não haver, em sua maioria, uma relação entre as diretrizes ou heurísticas de cada um, utilizadas no desenvolvimento e avaliação dos projetos. Apenas três das publicações relatadas, Mi et al. (2014), Leporini e Paternò (2003) e Alvarez, Jaramillo e Builes (2017), utilizam heurísticas de usabilidade destinadas a projetos acessíveis, todas desenvolvidas pelos próprios autores. O conjunto de Mi et al. (2014) é destinado especificamente a projetos de smartphones, enquanto a pesquisa de Leporini e Paternò (2003) atenta para o desenvolvimento e avaliação de websites para usuários que utilizam leitores de tela e Alvarez, Jaramillo e Builes (2017) para o desenvolvimento de softwares. Alguns autores destacam a importância da coordenação entre a usabilidade e a acessibilidade, reforçando que a conformidade com as diretrizes de acessibilidade não necessariamente conduz à usabilidade, e vice-versa. Percebe-se, em algumas publicações que atentaram para a usabilidade, a realização de testes com usuários, ao invés da avaliação heurística. É importante salientar, porém, que a utilização de heurísticas de usabilidade, de acordo com 
Rubin e Chisnell (2008), pode ser mais adequada em alguns casos, sendo mais efetiva em termos de custo, tempo dispendido e precisão do que o teste com usuários.

\section{CONCLUSÃO}

O presente artigo buscou analisar as heurísticas de usabilidade utilizadas em desenvolvimentos e avaliações voltados a pessoas com deficiência, de modo a investigar a ocorrência de heurísticas específicas destinadas a esse fim. Através da pesquisa, pôde-se ter um panorama da forma como a usabilidade é tratada em projetos destinados a esses indivíduos. Percebe-se uma grande preocupação dos autores com o atendimento às diretrizes de acessibilidade, sendo as heurísticas de usabilidade trabalhadas, usualmente, de forma independente, como requisitos distintos. Alguns autores preocupamse com o desenvolvimento de heurísticas específicas para usabilidade de projetos acessíveis, porém de forma pontual, ou seja, destinadas ao objeto de estudo da publicação em questão. O desenvolvimento de heurísticas de usabilidade voltadas à acessibilidade poderia garantir, em conformidade com as diretrizes de acessibilidade existentes, que as interfaces fossem, além de acessíveis a todos os usuários, fáceis de utilizar, oferecendo eficácia, eficiência e satisfação no desempenho das tarefas almejadas. O presente artigo pretende orientar estudos futuros que busquem a coordenação entre a usabilidade e a acessibilidade, podendo a pesquisa ser estendida no sentido de aprofundar a investigação de heurísticas de usabilidade voltadas a projetos acessíveis, e, possivelmente, resultar no desenvolvimento de um conjunto de heurísticas destinadas a este fim. 


\section{AGRADECIMENTOS}

O presente trabalho foi realizado com apoio da Coordenação de Aperfeiçoamento de Pessoal de Nível Superior - Brasil (CAPES) - Código de Financiamento 001.

\section{REFERÊNCIAS}

ABNT - ASSOCIAÇÃO BRASILEIRA DE NORMAS TÉCNICAS -. ISO 9241-11: requisitos ergonômicos para o trabalho com dispositivos de interação visual: parte 11: orientações sobre usabilidade. Rio de Janeiro: ABNT, 2002.

ALI, Saqib; ALBALUSHI, Taiseera; ALBADI, Ali. Guidelines and deployment of accessibility-aware framework approach. International Journal of Web Information Systems, [S. I.], v. 13, n. 2, p. 114-139, 2017.

ALVAREZ, Jhon Fernando Sánchez; JARAMILLO, Carlos Mario Zapata; BUILES, Jovani Alberto Jiménez. Evaluación heurística de la usabilidad de software para facilitar el uso del computador a personas en situación de discapacidad motriz. Revista EIA, Las Palmas, v. 14, n. 27, p. 63, 2017.

ANSARI, Rabab Fatima; BAQAR, Afifa; HASSAN, Hamna; SAEED, Fareeha. Heuristic, accessibility and usability evaluations of Pakistan's e-government websites. Electronic Government, an International Journal, Pensacola, v. 12, n. 1, p. 66, 2016. 
CONSTANTINE, Larry L.; LOCKWOOD, Lucy A. D. Software for use: a practical guide to the models and methods of usage-centered design. New York: Addison-Wesley Publishing, 1999.

CYBIS, Walter; BETIOL, Adriana Holtz; FAUST, Richard. Ergonomia e usabilidade: conhecimentos, métodos e aplicações. 3. ed. São Paulo: Novatec, 2015.

HARNISS, Mark; AMTMANN, Dagmar; COOK, Debbie; JOHNSON, Kurt. Considerations for developing interfaces for collecting patient-reported outcomes that allow the inclusion of individuals with disabilities. Medical Care, Philadelphia, v. 45, n. 5, suppl. 1, p. S48-S54, 2007.

KING, Bridgett A. King; YOUNGBLOOD, Norman E. E-government in Alabama: an analysis of county voting and election website content, usability, accessibility, and mobile readiness. Government Information Quarterly, Krems, v. 33, n. 4, p. 715-726, 2016.

LEPORINI, Barbara; PATERNÒ, Fabio. Criteria for usability of accessible web sites. In: CARBONEL, Nöelle; STEPHANIDIS, Constantine. (Eds.). Universal access: theorical perspectives, practice and experience. Heidelberg, BW: Springer, 2003. p. 43-55.

MI, Na; CAVUOTO, Lora A.; BENSON, Kenneth; SMITH-JACKSON, Tonya; NUSSBAUM, Maury A. A heuristic checklist for an accessible smartphone interface design. Universal Access in the Information Society, London, v. 13, n. 4, p. 351-365, 2014.

NIELSEN, Jakob. Usability engineering. San Francisco: Morgan Kaufman, 1994. NORMAN, Don. The design of everyday things. New York, NY: Basic Books, 2013.

PICKING, Rich; GROUT, Vic; MCGINN, John; CRISP, Jodi; GROUT, Helen. Simplicity, consistency, universality, flexibility and familiarity. IntSAernational Journal of Ambient Computing and Intelligence, [S. I.], v. 2, n. 3, p. 40-49, 2010.

RUBIN, Jeff; CHISNELL, Dana. Handbook of usability testing: how to plan, design, and conduct effective tests. 2. ed. Indianapolis: Wiley Publishing, Inc., 2008.

SCHMUTZ, Sven; SONDEREGGER, Andreas; SAUER, Juergen. Implementing recommendations from web accessibility guidelines: a comparative study of nondisabled users and users with visual impairments. Human Factors: the journal of the human factors and ergonomics society, New York, v. 59, n. 6, p. 956-972, 2017.

SHNEIDERMAN, B. Designing the user interface: strategies for effective human-computer interaction. 2. ed. MA: Co., Addison-Wesley Publ., 1999.

SHNEIDERMAN, Ben; PLAISANT, Catherine. Designing the user interface: strategies for effective human-computer interaction. 4. ed. Boston: Pearson Education, Inc., 2005. v. 215. 
STEWART, Ron; NARENDRA, Vivek. Accessibility and usability of online library databases. Library Hi Tech, Ann Arbor, v. 23, n. 2, p. 265-286, 2005.

YADRICH, Donna Macan; FITZGERALD, Sharon A.; WERKOWITCH, Marilyn; SMITH, Carol E. Creating patient and family education web sites: assuring accessibility and usability standards. CIN: computers informatics nursing, Hagerstown, v. 30, n. 1, p. 46-54, 2012.

Data de submissão: 2018-06-23

Data de aceite: 2018-10-11 